\section{Síndrome metabólico en pacientes con infección por VIH: ¿oportunidad para la suplementación nutricional?}

\section{Metabolic syndrome in HIV patients: an opportunity for nutritional supplementation?}

\section{Sr. Editor:}

La infección por el virus de la inmunodeficiencia humana (VIH) es una epidemia global, con especial repercusión en países en vías de desarrollo. Hasta el año 2016, se han reportado 36,7 millones de personas infectadas con el VIH en todo el mundo, de las cuales $46 \%$ residían en países de bajos y medianos ingresos. Desde la introducción del tratamiento anti-retroviral de gran actividad (TARGA) se ha visto una reducción marcada de la mortalidad y aumento en la calidad y esperanza de vida de estos pacientes ${ }^{1}$. No obstante, esta prolongación de los años de vida ha traído consigo la aparición de complicaciones crónicas metabólicas como el síndrome metabólico (SM) ${ }^{2}$.

Dentro de los componentes que conforman este síndrome se encuentran alteraciones en la presión arterial, glucosa plasmática, triglicéridos y colesterol HDL (lipoproteínas de alta densidad) séricos, y obesidad abdominal ${ }^{3}$. Asimismo, el SM es un factor de riesgo importante para el desarrollo de enfermedades cardiovasculares y cerebrovasculares ${ }^{4}$. En consecuencia, el manejo de sus componentes es relevante para la práctica médica y es un tópico de interés para la investigación clínica. Primariamente, se requeriría un abordaje integral en el tratamiento antihipertensivo, hipolipemiante y de las alteraciones del metabolismo de la glucosa ${ }^{5}$. Si bien las estrategias farmacológicas pudiesen ser una buena alternativa, se sabe que la polifarmacia disminuye la adherencia al TARGA -no sólo por el mayor número de medicamentos o tomas, sino también por los potenciales efectos adversos de los medicamentos- con la subsecuente disminución del efecto del TARGA en los resultados clínicos, y con la contribución a la diseminación de cepas del VIH resistentes ${ }^{6}$. Es por ello que, en los últimos años, se ha ampliado la búsqueda de otras alternativas terapéuticas no farmacológicas para el manejo del SM de estos pacientes, dentro de las cuales se encuentran las intervenciones nutricionales.

Entre las intervenciones nutricionales de primera línea está el cambio del estilo de vida, la que incluye una dieta hipocalórica asociada a actividad física. Sin embargo, se ha demostrado que este enfoque dietético puede ser insuficiente por distintas causas; como la falta de adherencia a esta intervención sobre todo en la población latina ${ }^{7}$. Por ello, han surgido alternativas de suplementación; como el uso de análogos de ácido nicotínico, los cuales reducen los niveles de triglicéridos y aumentan los niveles de
HDL sérico ${ }^{8,9}$; y la suplementación con cromo, la cual ha demostrado reducir la resistencia a la insulina y disminuir los niveles de triglicérido ${ }^{10}$. No obstante, debido a que la forma de presentación de estos suplementos también afectaría la adherencia, en los últimos años se ha ampliado la investigación de la suplementación con ácidos grasos $\Omega-3$, los cuales tienen distintos modos de presentación que hace fácil su consumo.

El uso de ácidos grasos $\Omega-3$ se ha convertido en una buena opción, ya que tienen un buen perfil de seguridad y se ha comprobado su efecto sobre algunos componentes del $\mathrm{SM}^{11}$. A pesar de ello, existe aún controversia sobre la eficacia del uso de suplementos con $\Omega-3$ ya que se han realizado diversos estudios sobre su efecto independiente sobre cada componente del SM; sin embargo, no existe evidencia de su efecto general sobre el síndrome. En vista de lo anterior, sería importante ampliar la investigación sobre el efecto de los ácidos grasos $\Omega-3$ en todos los componentes del SM. De esta manera, se tendrá en cuenta esta potencial alternativa de intervención nutricional en el manejo multidisciplinario del SM.

\section{Referencias bibliográficas}

1.- Organización Mundial de la Salud. Estrategia mundial del sector de la salud contra el VIH, 2016-2021. Suiza. 2016. http://apps.who.int/iris/bitstream/10665/250574/1/WHOHIV-2016.05-spa.pdf?ua=1.

2.- Paula A A, Falcão M C, Pacheco A G. Metabolic syndrome in HIV-infected individuals: underlying mechanisms and epidemiological aspects. AIDS Res Ther 2013; 10: 32.

3.- Executive summary of the third report of the national cholesterol education program (NCEP) expert panel on detection, evaluation, and treatment of high blood cholesterol in adults (Adult Treatment Panel III). JAMA 2001; 285: 2486-97.

4.- Mottillo S, Filion K B, Genest J, Joseph L, Pilote L, Poirier $\mathrm{P}$, et al. The metabolic syndrome and cardiovascular risk a systematic review and meta-analysis. J Am Coll Cardiol 2010; 56: 1113-32.

5.- Ford E S. Prevalence of the metabolic syndrome defined by the International Diabetes Federation among adults in the U.S. Diabetes Care 2005; 28: 2745-9.

6.- Gerenutti M, Martínez A M V, Bergamaschi C C. The Effectiveness of pharmaceutical care model on adherence to antiretroviral therapy: a SAME-based cohort study in Brazil. Adv Pharm Bull 2017; 7: 469-72.

7.- Stradling C, Chen Y F, Russell T, Connock M, Thomas $\mathrm{G} \mathrm{N}$, Taheri S. The effects of dietary intervention on HIV dyslipidaemia: a systematic review and meta-analysis. PLoS One 2012; 7: e38121.

8.- Hadigan C, Liebau J, Torriani M, Andersen R, Grinspoon S. Improved triglycerides and insulin sensitivity with 3 months of acipimox in human immunodeficiency virus-infected patients with hypertriglyceridemia. J Clin Endocrinol Metab 2006; 91: 4438-44.

9.- Chow D C, Stein J H, Seto T B, Mitchell C, 
Sriratanaviriyakul N, Grandinetti A, et al. Short-term effects of extended-release niacin on endothelial function in HIVinfected patients on stable antiretroviral therapy. AIDS 2010; 24: 1019-23.

10.- Aghdassi E, Arendt B M, Salit I E, Mohammed S S, Jalali P, Bondar $\mathrm{H}$, et al. In patients with HIV-infection, chromium supplementation improves insulin resistance and other metabolic abnormalities: a randomized, double-blind, placebo controlled trial. Current HIV Research 2010; 8: 11320.

11.- Viera A D S, Silveira G R M D. Effectiveness of n-3 fatty acids in treatment of hypertriglyceridemia in HIV/AIDS patients: a meta-analysis. Cien Saude Colet 2017; 22: 2659 -

69.

Wendy Valdivia-Caramantín, Julca-Malcay Edward Mezones-Holguín

Facultad de Ciencias de la Salud, Universidad Peruana de Ciencias Aplicadas.

Lima, Perú.

Correpondencia a: michellvc@gmail.com 